\title{
Optical characterization of strain sensing cables for Brillouin optical time domain analysis
}

\author{
Filippo Bastianini ${ }^{1}$, Francesco Falcetelli ${ }^{2}$, Pawet Bocheński ${ }^{3}$, Leonardo Rossi ${ }^{4}$, Raffaella Di Sante ${ }^{2}$, and Gabriele \\ Bolognini ${ }^{4}{ }^{*}$
}

${ }^{1}$ Sestosensor s.r.l., 140 via Gesso, 40069 Zola Predosa, Italy

${ }^{2}$ Dept. of Industrial Engineering, University of Bologna, Forlì, Italy

${ }^{3}$ Fibrain Sp. z.o.o., Wspólna 4A, Rzeszów 35-205, Poland

${ }^{4}$ Consiglio Nazionale delle Ricerche, IMM Institute, 101 via P. Gobetti, 40129 Bologna Italy

\begin{abstract}
Two innovative optical fibre cable layouts designed to improve BOTDA strain measurement accuracy through improved strain transfer efficiency are presented, discussed and tested through experiments, analytical and numerical modelling. The second improved design presents good features to minimize the mismatch between measured and actual strain.
\end{abstract}

\section{Introduction}

Optical fiber sensors represent one of the most interesting technologies for the monitoring of structures in many different fields. Among their many advantages compared to their electronic counterparts, optical fiber sensors are light weight, small size (which makes them suitable to be embedded in composites and other materials), immune to electromagnetic interference and capable of transmitting a large amount of information [1-7]. Among the various distributed sensing techniques, Brillouin optical time domain analysis (BOTDA) gained signficant relevanceamong the scientific and industrial community due to its capability, through the use of the Stimulated Brillouin Scattering (SBS) phenomenon, to monitor large structures by extracting temperature and strain profiles along tens of $\mathrm{km}$ of sensing fiber with spatial resolutions of the order of few centimetres [8-10]. While a great deal of study focuses on the interrogating system, an important factor for BOTDA sensors performance is the structure on the sensing cable. This is because in most applications, exposure to harsh environmental conditions makes it necessary for the sensing fiber to be protected by additional layers, but this can cause a mismatch between the strain at the core (the measured strain) and the strain in the outer cable sheath (the real strain) [11], which becomes even more significant. In this study, an experimental setup based on swept Swept Wavelength Interferometry (SWI) distributed strain sensing technique is used alongside analytical and numerical simulations to accurately evaluate the strain affecting sensing cables built from two new designs intended for BOTDA and is compared to the strain perceived by the structure they are attached to in order to evaluate their strain transfer efficiency.

\footnotetext{
* Corresponding author: bolognini@bo.imm.cnr.it
}

\section{Sensing cable design}

The two designs are shown in Fig. 1. The first sensing cable design was developed with the following goals as standard: (i) optimized mechanical coupling with the structure; (ii) protection against harsh environmental conditions; (iii) stable attenuation and sensitivity over the range of operational conditions; (iv) capability of withstanding large deformations; (v) absence of creep and other long-term phenomena capable of degrading the measuring performance of the cable. During preliminary testing, the performance of this design raised several concerns in terms of fiber uniformity, decoupling of the temperature compensation fiber and overall mechanical coupling between the strain sensing fiber and the observed structure. The second design, compared to the first one, was introduced as a way to address these issues with the First sensing cable design
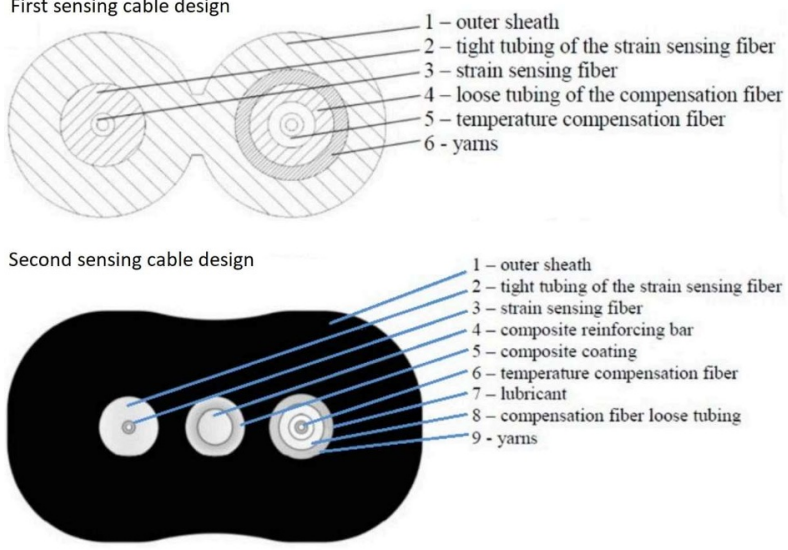

Fig. 1. Schematics of the cable prototypes.

following changes: (i) lower speed ramp-up during fabrication to ensure better uniformity of the fibre pre- 
stress; (ii) the presence of a silane-based lubricant to reduce the drag on the temperature compensation fiber; (iii) different cable jacket material to avoid slippage with the inner fibre tubing; (iv) structured surface to improve the mechanical coupling with the host structure. These changes are expected to improve overall sensing performance in terms of strain sensing accuracy, better distinction with temperature readings, and more reliable strain transfer.

\section{Testing Methods}

First, the performance of the devices was evaluated through numerical simulation based on the development of a Finite Element Method (FEM) model of the two cables using Abaqus/CAE, in which every single layer of the cables was taken into account. The shape of the cables was also accurately reproduced based on direct observation of the cable cross section via microscope. Between every layer was assumed to be present a perfect bonding which translated in applying a tie constraint between their surfaces. The behavior of the cables under different loads was simulated by imposing fixed displacements in the substrate structure, thus reproducing real experimental conditions.

Afterwards, the strain transfer of the two cables was experimentally assensed through the use of an Optical Backscatter Reflectometer (OBR) based on SweptWavelength Interferometry (SWI) [12-14]. SWI involves measuring the Rayleigh backscattered signal generated by refractive index variations in silica fibers. For each point of the fiber, the strain value can be derived from the reflected wavelength $\lambda_{R}$ by treating the fiber as a Fiber Bragg Grating (FBG) sensor. While the change in refractive index is generally nonperiodical, it is possible to define a local average perturbation period $\Lambda_{\mathrm{AVG}}$ dependent on $\lambda_{R}$ and the effective refractive index $n_{\text {eff }}$ through the relation:

$$
\lambda_{R}=2 n_{e f f} \Lambda_{A V G}
$$

By detecting the Rayleigh backscatter before the experiment, it is possible to determine a stable baseline. When a certain point of the fiber is subject to strain or temperature variations, the corresponding reflected wavelength in the trace shifts from the baseline by an amount $\Delta \lambda_{R}$ defined by the relation:

$$
\frac{\Delta \lambda_{R}}{\lambda_{R}}=-\frac{\Delta v_{R}}{v_{R}}=K_{\varepsilon} \varepsilon+K_{T} \Delta T
$$

Where $K_{\varepsilon}$ and $K_{T}$ are the strain and temperature coefficients, respectively, while $v_{R}$ and $\Delta v_{R}$ are the corresponding reference frequency and frequency shift. In order to completely exclude the contribution $K_{T} \Delta T$ caused by temperature variation, all experiments in this work were performed at constant ambient temperature to filter any undesired thermal evolution, and thus the temperature term can be considered negligible. For what concerns the strain term $K_{\varepsilon} \varepsilon$, the strain coefficient $K_{\varepsilon}$ is defined as:

$$
K_{\varepsilon}=1-\rho_{\varepsilon}
$$

Where $\rho_{\varepsilon}$ is the photoelastic coefficient, which is usually calculated with the following formula:

$$
\rho_{\varepsilon}=\frac{n_{e f f}^{2}}{2}\left[p_{12}-v\left(p_{11}+p_{12}\right)\right]
$$

Where $p_{11}$ and $p_{12}$ are the components of the strain optic tensor, while $v$ is Poisson's ratio [15]. For standard germanium doped silica fibers, like the ones used in this work, the photoelastic coefficient is known to be equal for 0.22 . As a result, the strain coefficient should be equal to 0.78. Knowing these parameters and the central wavelength $\bar{\lambda}$ of the scanning process, it is possible to determine the relation between the strain and the frequency shift as a linear relation of the form:

$$
\varepsilon=-\frac{\bar{\lambda}}{c K_{\varepsilon}} \Delta v_{R}=\alpha \Delta v_{R}
$$

From this formula, assuming $\bar{\lambda}=1306 \mathrm{~nm}$ for this measurement, the value for $\alpha$ is found to be $-5.59 \mu \varepsilon / \mathrm{GHz}$. While this value may be realistic, it is still affected by variable factors such as dopant species concentration in the fiber core and outer layer composition (including cladding and coating) which all contribute to the uncertainty of the estimation [16]. Because of this, the strain coefficient was ultimately found through an experimental calibration process, which involved inserting the fibers in a test rig employing a highprecision linear actuator with a travel range of $500 \mathrm{~mm}$ and a resolution of $0.02 \mathrm{~mm}$, allowing for characterization of up to $3.5 \mathrm{~m}$ of fibers. The frequency displacement was measured while the fiber was subject to different strain values, ranging from 0 to $4000 \mu \varepsilon$, with a step of $100 \mu \varepsilon$. The results of the calibration for both cable design are shown in Fig. 2. From linear fitting, the $\alpha$ parameter, which was the inverse of the slope, was determined to be $\alpha_{1}=-5.520 \pm 0.018 \mu \varepsilon / \mathrm{GHz}$ for the first cable design and $\alpha_{2}=-5.590 \pm 0.023 \mu \varepsilon / \mathrm{GHz}$ for the second one.
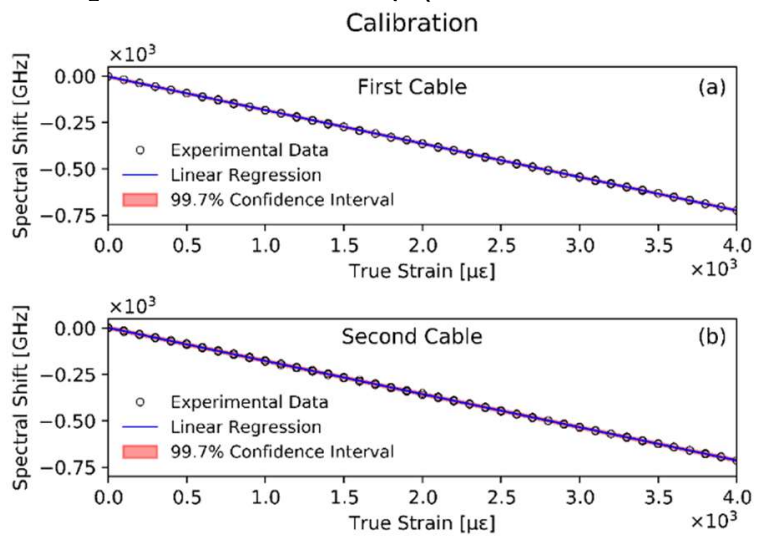

Fig. 2. Calibration data for the first (a), and the second (b), cable prototypes.

Once the calibration process was complete, SWI could be used to evaluate the strain transfer properties of the two fiber design. For the experimental evaluation, the sensing fibers were bonded through epoxy adhesive (LOCTITE ${ }^{\circledR}$ 
EA $9466^{\mathrm{TM}}$ ) on the surface of an aluminium specimen, which in turn was put into a tensile strength machine, as shown in Fig. 3. Its dimensions were $300 \mathrm{~mm}$ in length, 8 $\mathrm{mm}$ in thickness and $20 \mathrm{~mm}$ width, a Young modulus of $71.7 \mathrm{GPa}$ and a shear modulus of $26.9 \mathrm{GPa}$. Sensing cables built according to the first and second design were attached at the opposite sides of the specimen, in order not to come in contact with the clamps of the tensile strain machine, which would have added unwanted bending radii. The bonding length between the cable and the specimen required for a complete strain transfer was determined from the preliminary results of the previously showcased numerical model, which found optimal results for $270 \mathrm{~mm}$.

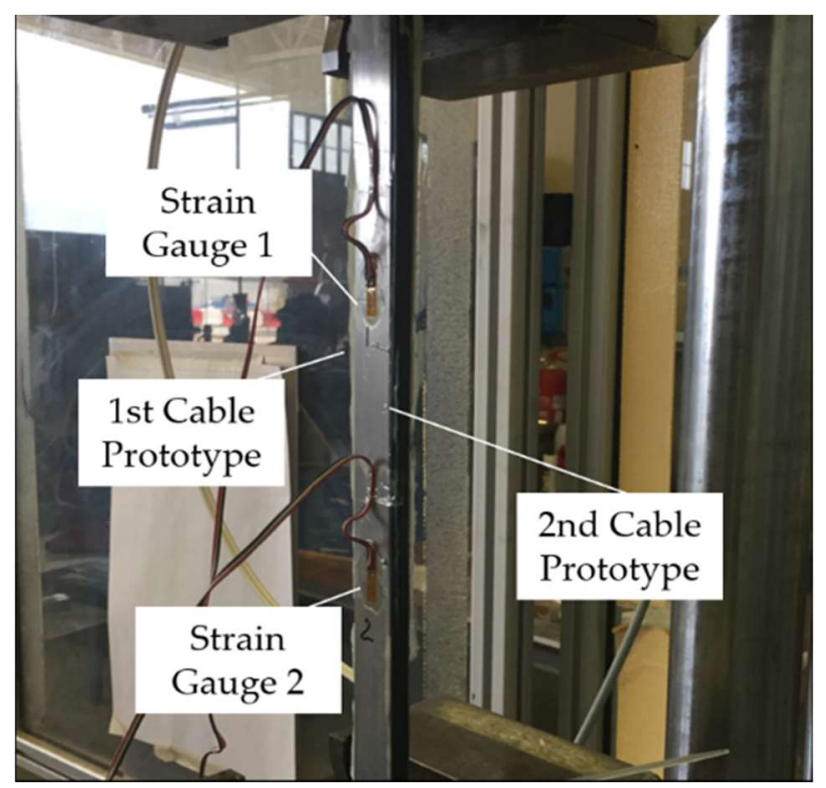

Fig. 3. Aluminium specimen in the tensile strength machine.

During the experiment, the aluminium specimen was subjected to loads ranging from $5 \mathrm{kN}$ to $25 \mathrm{kN}(5,10,15$

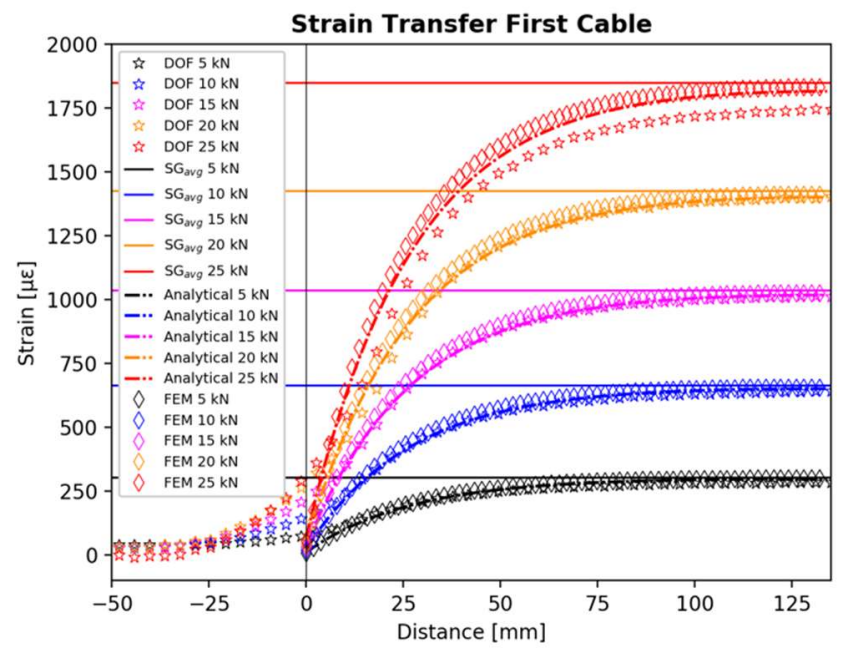

20 and $25 \mathrm{kN}$ ). Two electrical strain gauges were fixed on the opposite ends of the specimen's length and used as a reference to measure the effective longitudinal strain value of the specimen. In particular, the average of their measurement was used as the asymptotic strain value that should be detected by the two sensing cables.

As further validation, the experimental results were compared with a known analytical model for strain transfer developed in [17], represented by the following formula:

$$
\varepsilon_{f}(x)=\varepsilon_{h}\left[1-\frac{\cosh (k x)}{\cosh (k L)}\right]
$$

In which $\varepsilon_{f}$ represents the strain profile of the fiber core, $\varepsilon_{h}$ the strain profile in the host structure, $L$ denotes the bonding length and $x$ represents the spatial coordinate along the fiber axis. Finally, $k$ corresponds to the shear lag parameter, and is the fundamental variable of the model, enclosing all the geometrical and mechanical aspect of the sensing cable and its interface with the structure. In particular, the higher the value, the more efficiently the strain is transferred from the structure to the sensing cable. While in principle its value can be derivable a priori from the properties of the sensing cable, it is commonly considered more feasible to tune the value based on experimental data in order to correct the initial guess for the value.

\section{Results}

The results are summarized in Fig. 4. Due to the symmetrical nature of the experiment, only one half of the strain profile is plotted (the first $135 \mathrm{~mm}$ of bonding length). The 0 point in the distance axis (the vertical line in both plots) is chosen as the first bonding point between the cable and the aluminium specimen. The horizontal solid lines correspond to the reference strain value obtained by the the two strain gauges for each load case.

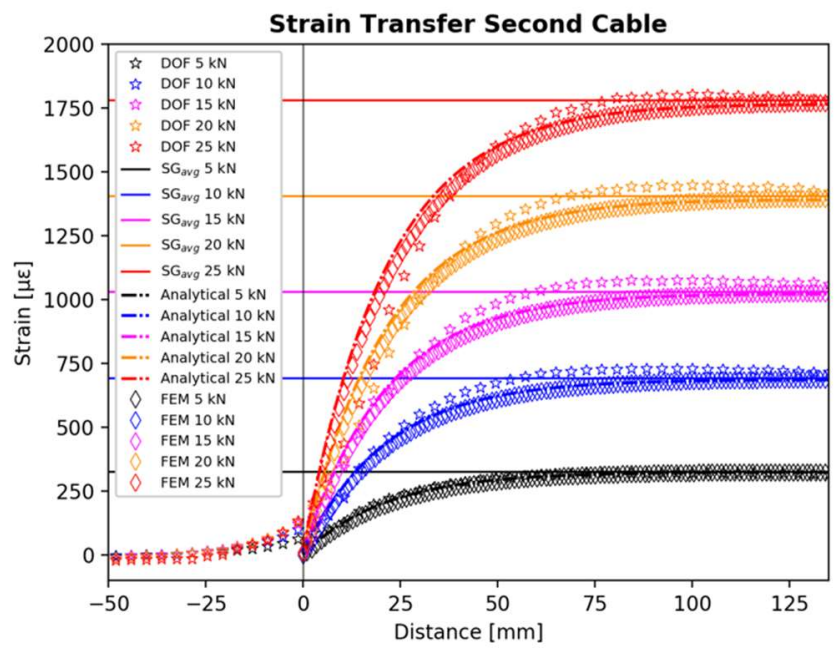

Fig. 4. Strain transfer of the first sensing cable (left) and second sensing cable (right). Horizontal lines: strains measured by the gauges. Stars: OBR measurements. Dashed lines: analytical model. Diamonds: numerical FEM model. 
The diamond shaped symbols are the results obtained from the FEM numerical model. The SWI sensing measurements are represented by star symbols and are denoted in the legend with the DOF acronym. Finally, the dashed lines are the strain profiles predicted by the strain transfer model. The strain transfer improvement from the first sensing cable with respect to the second can be easily seen at the highest load levels, where even in the midline $(\mathrm{x}=135 \mathrm{~mm})$, there is a mismatch between the strain of the first sensing cable and the strain of the gauge, representing an incomplete strain transfer. On the other hand, the second developed sensing cable is always capable to reach the strain level measured by the two strain gauges, proving it as a viable alternative for BOTDA sensing cables.

In addition, for both designs it can be seen that there is a consistent overlap between the experimentally obtained strain values for the sensing cable and the one predicted with the numerical and theoretical models, showing how both these tools can allow to make reliable predictions without the need of performing experiments, although both systems still improved thanks to experimental data.

\section{Conclusion}

In this work, a sensing method based on swept wavelength interferometry has been used to evaluate two newly manufactured optical fibre temperature and strain sensing cables. In particular, these cables are intended to be employed in Brillouin optical time domain analysis to extract temperature and strain spatial distributions in large structures at centimetre level spatial resolution. Of the two cable designs, the first one was intended to satisfy a series of requirements for effective strain sensing performance, but a series of construction issues caused it to have non optimal strain transfer capabilities and in general issues that are expected to prevent it from operating at optimal operating conditions, leading to the realization of the second design in order to solve them. Besides swept wavelength interferometry, the two designs were also evlauated through numerical and analytical approaches. The results showed a good agreement between the experimental methods and both numerical and analytical models, showing that swept wavelength interferometry is an effective method for characterizing the strain transfer behaviour of optical sensing cables and that the numerical and analytical models can be considered reliable tools to make predictions. As expected, these results showed that the second design presented superior strain transfer capabilities, always reaching the same strain value of the actual specimen, proving it to be a viable alternative for BOTDA sensing. The first design, on the other hand, due to the issue detailed in this work, presented a mismatch between the strain detected by the cable and the one effectively applied to the specimen, especially at higher strain values.

This activity has received funding from the European Union through Horizon 2020, the Framework Programme for Research and Innovation, under project SLAM-DAST, Grant Agreement No. 971149.

\section{References}

1. R. Di Sante, Sensors, 15, 18666-18713 (2015).

2. D. Marini, M. Iuliano, F. Bastianini, G. Bolognini, J. Light. Technol., 36, 1131-1137 (2018)

3. M.A. Soto, P.K. Sahu, S. Faralli, G. Sacchi, G. Bolognini, F. Di Pasquale, B. Nebendahl, C. Rueck, Proceedings of SPIE, 66193B (2017)

4. F. Falcetelli, L. Rossi, R. Di Sante, G. Bolognini, Sensors. 20, 3100 (2020).

5. D. Marini, L. Rossi, F. Bastianini, G. Bolognini, 20th Italian National Conference on Photonic Technologies (Fotonica 2018), CP748, 147646 (2018)

6. L. Rossi, D. Marini, F. Bastianini, G. Bolognini, Opt. Express, 27, 29448-29460 (2019).

7. G. Bolognini, S. Faralli, A. Chiuchiarelli, F. Falconi, F. Di Pasquale, IEEE Photonics Technol. Lett., 18, 1591-1593 (2006).

8. M.A. Soto, M. Taki, G. Bolognini, F. Di Pasquale, Opt. Express, 20, 6860-6869 (2012).

9. A. H. Hartog, An introduction to distributed optical fibre sensors, 1st ed., CRC press, 2017.

10. D. Marini, L. Rossi, F. Bastianini, G. Bolognini, OSA Technical Digest, ThE71, 140688 (2018).

11. F. Bastianini, R. Di Sante, F. Falcetelli, D. Marini, G. Bolognini, Sensors, 19, 5172 (2019).

12. E. D. Moore, R. R. McLeod, Opt. Express, 16, 1313913149 (2008)

13. D.K. Gifford, S.T. Kreger, A.K. Sang, M.E. Froggatt, R.G. Duncan, M.S. Wolfe, B.J. Soller, Proceedings of the Fiber Optic Sensors and Applications V, 6770, 67700F (2007).

14. B.J. Soller, M.S. Wolfe, M.E. Froggat, Proceedings of the OFC Technical Digest; NWD3 (2005).

15. Rao, Y.J. Fiber Bragg grating sensors: principles and applications. In Optical Fiber Sensor Technology; Grattan, K.T.V., Meggitt, B.T. (Eds.; Springer US: Boston, MA, 1998), pp. 355-379.

16. LUNA Technologies Optical Backscatter Reflectometer User Guide (2009).

17. D. Li, H. Li, L. Ren, G. Song, Opt. Eng, 45, 024402 (2006).

\footnotetext{
Corresponding author: bolognini@bo.imm.cnr.it
} 